\title{
A fast butterfly algorithm for the hyperbolic Radon transform
}

Jingwei Hu*, Sergey Fomel, The University of Texas at Austin, Laurent Demanet, Massachusetts Institute of Technology, and Lexing Ying, The University of Texas at Austin

\section{SUMMARY}

We introduce a fast butterfly algorithm for the hyperbolic Radon transform commonly used in seismic data processing. For two-dimensional data, the algorithm runs in complexity $O\left(N^{2} \log N\right)$, where $N$ is representative of the number of points in either dimension of data space or model space. Using a series of examples, we show that the proposed algorithm is significantly more efficient than conventional integration.

\section{INTRODUCTION}

In seismic data processing, the Radon transform (RT) (Radon 1917) is a set of line integrals that maps mixed and overlapping events in seismic gathers to a new transformed domain where they can be separated (Gardner and Lu, 1991). The line integrals can follow different curves; straight lines (linear RT or slant stack), parabolas (parabolic RT), or hyperbolas (hyperbolic RT or velocity stack) are most commonly used. A major difference between these transforms is that the former two are time-invariant whereas the latter is time-variant. When the curves are time-invariant, the transform can be performed efficiently in the frequency domain by the Fourier transform shift theorem. On the contrary, the hyperbolic Radon transform has to be computed in the time domain, which is not feasible in general due to the large size of seismic data. Nevertheless, the hyperbolic transform is often preferred as it better matches the true seismic events. Based on the special properties of the Radon operator, many approaches were proposed to speed up the computation in the time domain (Thorson and Claerbout 1985: Sacchi, 1996, Cary, 1998, Trad et al. 2002).

In this work, we construct a fast butterfly algorithm to efficiently evaluate the hyperbolic Radon transform. As opposed to the conventional, expensive velocity scan (i.e., direct integration + interpolation), our method provides an accurate approximation in only $O\left(N^{2} \log N\right)$ operations for 2D data. Here $N$ depends only on the range of the parameters and can often be chosen small compared to the problem size. The adjoint of the hyperbolic transform can be implemented similarly without extra difficulty.

The Radon transform has been widely used to separate and attenuate multiple reflections (Hampson, 1986; Yilmaz [1989. Foster and Mosher, 1992; Herrmann et al., 2000, Moore and Kostov 2002, Hargreaves et al. 2003, Trad, 2003). By introducing the fast solver, our hope is to improve the inversion process either iteratively or directly.

The rest of the paper is organized as follows. We first introduce the low-rank approximation and the butterfly structure; then using these building elements, we construct our fast algorithm. Numerical examples of both synthetic and field data are presented next to illustrate the accuracy and efficiency of the proposed algorithm.

\section{ALGORITHM}

Assume $d(t, h)$ is a function in the data domain, then a hyperbolic Radon transform $R$ maps $d$ to function $(R d)(\tau, p)$ in the model domain (Thorson and Claerbout, 1985),

$$
(R d)(\tau, p)=\int d\left(\sqrt{\tau^{2}+p^{2} h^{2}}, h\right) d h
$$

Here $t$ is the time, $h$ is the offset, $\tau$ is the intercept, and $p$ is the slowness. Fixing $(\tau, p)$, the hyperbola $t=\sqrt{\tau^{2}+p^{2} h^{2}}$ describes the traveltime for the event; hence integration along these curves can be used to identify different reflections.

We adopt a different point of view to construct the algorithm by reformulating the transform (1) as a double integral,

$$
(R d)(\tau, p)=\iint \hat{d}(\omega, h) e^{2 \pi i \omega \sqrt{\tau^{2}+p^{2} h^{2}}} d \omega d h,
$$

where $\hat{d}(\omega, h)$ is the Fourier transform of $d(t, h)$ in the $t$ variable. Discretizing 2 in the $(\omega, h)$ domain, one obtains

$$
(R d)(\tau, p)=\sum_{\omega, h} e^{2 \pi i \omega \sqrt{\tau^{2}+p^{2} h^{2}}} \hat{d}(\omega, h) .
$$

For simplicity, we first perform a linear transformation to map $(\tau, p)$ to $\mathbf{x}=\left(x_{1}, x_{2}\right) \in X=[0,1]^{2}$, and $(\omega, h)$ to $\mathbf{k}=\left(k_{1}, k_{2}\right) \in$ $K=[0,1]^{2}: \tau=\left(\tau_{\max }-\tau_{\min }\right) x_{1}+\tau_{\min }, p=\left(p_{\max }-p_{\min }\right) x_{2}+$ $p_{\min } ; \omega=\left(\omega_{\max }-\omega_{\min }\right) k_{1}+\omega_{\min }, h=\left(h_{\max }-h_{\min }\right) k_{2}+$ $h_{\text {min }}$. If we define input (source) $f(\mathbf{k})=\hat{d}\left(\omega\left(k_{1}\right), h\left(k_{2}\right)\right)$, output (target) $u(\mathbf{x})=(R d)\left(\tau\left(x_{1}\right), p\left(x_{2}\right)\right)$, and the phase function $\Phi(\mathbf{x}, \mathbf{k})=\omega\left(k_{1}\right) \sqrt{\tau\left(x_{1}\right)^{2}+p\left(x_{2}\right)^{2} h\left(k_{2}\right)^{2}}$, then 3 becomes

$$
u(\mathbf{x})=\sum_{\mathbf{k} \in K} e^{2 \pi i \Phi(\mathbf{x}, \mathbf{k})} f(\mathbf{k}), \quad \mathbf{x} \in X
$$

which falls into the general discretized form of Fourier integral operators. Our algorithm for computing the summation in equation (4) follows that of Candès et al. (2009). Readers are referred there for detailed mathematical exposition.

\section{Low-rank approximations}

The magnitude of the phase $\Phi(\mathbf{x}, \mathbf{k})$ determines the degree of oscillation of the kernel $e^{2 \pi i \Phi(\mathbf{x}, \mathbf{k})}$. Let $N$ be an integer power of two, which is on the order of the maximum of $|\Phi(\mathbf{x}, \mathbf{k})|$ for $\mathbf{x} \in X$ and $\mathbf{k} \in K$. The exact choice of $N$ depends on the desired efficiency and accuracy of the algorithm, and several examples will be given in the numerical results. The design of the fast algorithm relies on the key observation that this kernel, properly restricted to subdomains of $\mathbf{x}$ and $\mathbf{k}$, admits accurate and low-rank separated approximations; i.e., if $A$ and $B$ are two 


\section{Fast Radon Transform}

square boxes in $X$ and $K$, with sidelengths $w(A), w(B)$ obeying $w(A) w(B) \leq 1 / N$, then

$$
e^{2 \pi i \Phi(\mathbf{x}, \mathbf{k})} \approx \sum_{t=1}^{r_{\varepsilon}} \alpha_{t}^{A B}(\mathbf{x}) \beta_{t}^{A B}(\mathbf{k}), \quad \mathbf{x} \in A, \mathbf{k} \in B,
$$

where the number $r_{\varepsilon}$ of expansions is independent of $N$ for fixed error $\varepsilon$. Furthermore, it is shown that this low-rank approximation can be constructed by a tensor-product Chebyshev interpolation of $e^{2 \pi i \Phi(\mathbf{x}, \mathbf{k})}$ in the $\mathbf{x}$ variable when $w(A) \leq$ $1 / \sqrt{N}$ and in the k variable when $w(B) \leq 1 / \sqrt{N}$. Specifically, when $w(B) \leq 1 / \sqrt{N}, \alpha_{t}^{A B}$ and $\beta_{t}^{A B}$ are given by

$$
\begin{aligned}
& \alpha_{t}^{A B}(\mathbf{x})=e^{2 \pi i \Phi\left(\mathbf{x}, \mathbf{k}_{t}^{B}\right)}, \\
& \beta_{t}^{A B}(\mathbf{k})=e^{-2 \pi i \Phi\left(\mathbf{x}_{\mathbf{0}}(A), \mathbf{k}_{t}^{B}\right)} L_{t}^{B}(\mathbf{k}) e^{2 \pi i \Phi\left(\mathbf{x}_{\mathbf{0}}(A), \mathbf{k}\right)} ;
\end{aligned}
$$

when $w(A) \leq 1 / \sqrt{N}, \alpha_{t}^{A B}$ and $\beta_{t}^{A B}$ are given by

$$
\begin{aligned}
& \alpha_{t}^{A B}(\mathbf{x})=e^{2 \pi i \Phi\left(\mathbf{x}, \mathbf{k}_{0}(B)\right)} L_{t}^{A}(\mathbf{x}) e^{-2 \pi i \Phi\left(\mathbf{x}_{t}^{A}, \mathbf{k}_{0}(B)\right)}, \\
& \beta_{t}^{A B}(\mathbf{k})=e^{2 \pi i \Phi\left(\mathbf{x}_{t}^{A}, \mathbf{k}\right)} .
\end{aligned}
$$

Here $\mathbf{x}_{\mathbf{0}}(A)$ and $\mathbf{k}_{\mathbf{0}}(B)$ denote the center of the boxes $A$ and $B$. $L_{t}^{B}(\mathbf{k})$ is the 2D Lagrange interpolation on the Chebyshev grid $\mathbf{k}_{t}^{B}$ :

$$
L_{t}^{B}(\mathbf{k})=\left(\prod_{s_{1}=0, s_{1} \neq t_{1}}^{q_{k_{1}}-1} \frac{k_{1}-k_{s_{1}}^{B}}{k_{t_{1}}^{B}-k_{s_{1}}^{B}}\right)\left(\prod_{s_{2}=0, s_{2} \neq t_{2}}^{q_{k_{2}}-1} \frac{k_{2}-k_{s_{2}}^{B}}{k_{t_{2}}^{B}-k_{s_{2}}^{B}}\right),
$$

with $\mathbf{k}_{t}^{B}=\left\{\left(k_{t_{1}}^{B}, k_{t_{2}}^{B}\right) \mid \mathbf{k}_{0}(B)+w(B)\left(z_{i_{1}}, z_{i_{2}}\right), 0 \leq i_{1} \leq q_{k_{1}}-\right.$ $\left.1,0 \leq i_{2} \leq q_{k_{2}}-1, r_{\varepsilon}=q_{k_{1}} q_{k_{2}}\right\}$, and $z_{i}=\frac{1}{2} \cos \left(\frac{i \pi}{q-1}\right)$ is the 1D Chebyshev grid of order $q$ on $[-1 / 2,1 / 2] . L_{t}^{A}(\mathbf{x})$ and $\mathbf{x}_{t}^{A}$ are defined accordingly.

\section{Butterfly structure}

To realize the above idea, the butterfly algorithm (Michielssen and Boag, 1996, O'Neil and Rokhlin 2007) turns out to be an appropriate tool. The main data structure underlying the algorithm is a pair of dyadic trees $T_{X}$ and $T_{K}$. The tree $T_{X}$ has $X=[0,1]^{2}$ as its root box (level 0 ) and is built by recursive, dyadic partitioning of $X$ until level $L=\log N$, where the finest boxes are of sidelength $1 / N$. The tree $T_{K}$ is built similarly but in the opposite direction. Figure 1 shows such a partition for $N=4$. A crucial property of this structure is that at arbitrary level $l$, the sidelengths of a box $A$ in $T_{X}$ and a box $B$ in $T_{K}$ always satisfy $w(A) w(B)=1 / N$. Thus, a low-rank approximation of the kernel $e^{2 \pi i \Phi(\mathbf{x}, \mathbf{k})}$ is available.

\section{Fast butterfly algorithm}

Our goal is to approximate the partial sum generated by the sources $\mathbf{k}$ inside any fixed box $B: u^{B}(\mathbf{x}):=\sum_{\mathbf{k} \in B} e^{2 \pi i \Phi(\mathbf{x}, \mathbf{k})} f(\mathbf{k})$.

1. Initialization. At level $l=0$, let $A$ be the root box of $T_{X}$. For each leaf box $B \in T_{K}$, equations 5 , 6 are valid since $w(B) \leq$ $1 / \sqrt{N}$. Then for $\mathbf{x} \in A, u^{B}(\mathbf{x}) \approx \sum_{t=1}^{r_{\varepsilon}} e^{2 \pi i \Phi\left(\mathbf{x}, \mathbf{k}_{t}^{B}\right)} \delta_{t}^{A B}$, where $\delta_{t}^{A B}:=\sum_{\mathbf{k} \in B} \beta_{t}^{A B}(\mathbf{k}) f(\mathbf{k})$ is given by

$$
\delta_{t}^{A B}=e^{-2 \pi i \Phi\left(\mathbf{x}_{0}(A), \mathbf{k}_{t}^{B}\right)} \sum_{\mathbf{k} \in B}\left(L_{t}^{B}(\mathbf{k}) e^{2 \pi i \Phi\left(\mathbf{x}_{0}(A), \mathbf{k}\right)} f(\mathbf{k})\right) .
$$


Figure 1: Butterfly structure for the special case of $N=4$.

Due to the special form of $\alpha_{t}^{A B}, \delta_{t}^{A B}$ can be treated as equivalent sources located at $\mathbf{k}_{t}^{B}$. We next aim at updating $\delta_{t}^{A B}$ until the end level $L$. This is done as follows.

2. Recursion. At $l=1,2, \ldots, L / 2$, for each pair $(A, B)$, let $A_{p}$ be $A$ 's parent and $B_{c}, c=1,2,3,4$ be $B$ 's children (see Figure 1). For each child, we have available from the previous level an approximation of the form

$$
u^{B_{c}}(\mathbf{x}) \approx \sum_{t^{\prime}=1}^{r_{\varepsilon}} e^{2 \pi i \Phi\left(\mathbf{x}, \mathbf{k}_{t^{\prime}}^{B_{c}}\right)} \delta_{t^{\prime}}^{A_{p} B_{c}}, \quad \text { for } \mathbf{x} \in A_{p} .
$$

Summing over all children gives

$$
u^{B}(\mathbf{x}) \approx \sum_{c=1}^{4} \sum_{t^{\prime}=1}^{r_{\varepsilon}} e^{2 \pi i \Phi\left(\mathbf{x}, \mathbf{k}_{t^{\prime}}^{B_{c}}\right)} \delta_{t^{\prime}}^{A_{p} B_{c}}, \quad \text { for } \mathbf{x} \in A_{p}
$$

Since $A \subset A_{p}$, this is of course true for any $\mathbf{x} \in A$. On the other hand, $e^{2 \pi i \Phi(\mathbf{x}, \mathbf{k})}$ also has a low-rank approximation of equivalent sources at the current level,

$$
u^{B}(\mathbf{x}) \approx \sum_{t=1}^{r_{\varepsilon}} e^{2 \pi i \Phi\left(\mathbf{x}, \mathbf{k}_{t}^{B}\right)} \delta_{t}^{A B}, \quad \text { for } \mathbf{x} \in A
$$

An easy calculation suggests

$$
\delta_{t}^{A B}=\sum_{c=1}^{4} \sum_{t^{\prime}=1}^{r_{\varepsilon}} \beta_{t}^{A B}\left(\mathbf{k}_{t^{\prime}}^{B_{c}}\right) \delta_{t^{\prime}}^{A_{p} B_{c}}
$$

Substituting $\beta_{t}^{A B}$ in 6 yields

$$
\delta_{t}^{A B}=e^{-2 \pi i \Phi\left(\mathbf{x}_{0}(A), \mathbf{k}_{t}^{B}\right)} \sum_{c=1}^{4} \sum_{t^{\prime}=1}^{r_{\varepsilon}}\left(L_{t}^{B}\left(\mathbf{k}_{t^{\prime}}^{B_{c}}\right) e^{2 \pi i \Phi\left(\mathbf{x}_{0}(A), \mathbf{k}_{t^{\prime}}^{B_{c}}\right)} \delta_{t^{\prime}}^{A_{p} B_{c}}\right) .
$$




\section{Fast Radon Transform}

3. Switch. A switch of the representation to 7,8 is needed at $l=L / 2$ since 5.67 is no longer valid as $l>L / 2$. Indeed, we can set

$$
\delta_{t}^{A B}=\sum_{s=1}^{r_{\varepsilon}} e^{2 \pi i \Phi\left(\mathbf{x}_{t}^{A}, \mathbf{k}_{s}^{B}\right)} \delta_{s}^{A B},
$$

where $\left\{\delta_{t}^{A B}\right\}$ denotes the new set of coefficients and $\left\{\delta_{s}^{A B}\right\}$ the old set.

4. Recursion. The rest of the recursion is analogous. For $l=L / 2+1, \ldots, L$, we have

$$
\delta_{t}^{A B}=\sum_{c=1}^{4} \sum_{t^{\prime}=1}^{r_{\varepsilon}} \alpha_{t^{\prime}}^{A_{p} B_{c}}\left(\mathbf{x}_{t}^{A}\right) \delta_{t^{\prime}}^{A_{p} B_{c}}
$$

Substituting $\alpha_{t}^{A B}$ in 77 gives

$$
\delta_{t}^{A B}=\sum_{c=1}^{4} e^{2 \pi i \Phi\left(\mathbf{x}_{t}^{A}, \mathbf{k}_{0}\left(B_{c}\right)\right)} \sum_{t^{\prime}=1}^{r_{\varepsilon}}\left(L_{t^{\prime}}^{A_{p}}\left(\mathbf{x}_{t}^{A}\right) e^{-2 \pi i \Phi\left(\mathbf{x}_{t^{\prime}}^{A_{p}}, \mathbf{k}_{0}\left(B_{c}\right)\right)} \delta_{t^{\prime}}^{A_{p} B_{c}}\right) .
$$

5. Termination. Finally we reach $l=L$, and $B$ is the entire domain $K$. For each $\mathbf{x} \in A$,

$$
u(\mathbf{x})=u^{B}(\mathbf{x}) \approx \sum_{t=1}^{r_{\varepsilon}} \alpha_{t}^{A B}(\mathbf{x}) \delta_{t}^{A B} .
$$

We thus set (after plugging in $\alpha_{t}^{A B}$ in 7 )

$u(\mathbf{x})=e^{2 \pi i \Phi\left(\mathbf{x}, \mathbf{k}_{0}(B)\right)} \sum_{t=1}^{r_{\varepsilon}}\left(L_{t}^{A}(\mathbf{x}) e^{-2 \pi i \Phi\left(\mathbf{x}_{t}^{A}, \mathbf{k}_{0}(B)\right)} \delta_{t}^{A B}\right), \quad \mathbf{x} \in A$.

\section{Discussion}

The main workload for the fast butterfly algorithm is in steps 2 and 4 . For each level, there are $N^{2}$ pairs of boxes $(A, B)$, and the operations between each $A$ and $B$ is a constant small number (depends on $r_{\varepsilon}$ ). Since there $\operatorname{are} \log N$ levels, the total cost is $O\left(N^{2} \log N\right)$. It is not difficult to see that step 3 takes $O\left(N^{2}\right)$, and steps 1 and 5 take $O\left(N_{\omega} N_{h}\right)$ and $O\left(N_{\tau} N_{p}\right)$ operations. Considering the initial Fourier transform of preparing data in the $(\omega, h)$ domain, we conclude that the overall complexity of the algorithm is roughly $O\left(N_{t} N_{h} \log N_{t}+C\left(r_{\varepsilon}\right) N^{2} \log N+\right.$ $\left.N_{\omega} N_{h}+N_{\tau} N_{p}\right)$. Normally $N_{\omega}=N_{t}$, but by symmetry and compactness of the Fourier transform $\hat{d}(\omega, h)$ (typical for most seismic data), we can significantly shorten the domain for $\omega$, hence further reduce the computational load.

In comparison, the conventional velocity scan requires at least $\mathrm{O}\left(N_{\tau} N_{p} N_{h}\right)$ computations, which quickly become a bottleneck as the problem size increases. Yet the efficiency of our algorithm is mainly controlled by $O\left(N^{2} \log N\right)$, where $N$ is roughly determined by the degree of oscillation of $e^{2 \pi i \omega \sqrt{\tau^{2}+p^{2} h^{2}}}$, i.e., the range of $\tau, p, h$, and $\omega$. In practice, $N$ can often be chosen smaller than $N_{\tau}, N_{p}, N_{h}$, and $N_{\omega}$.

There is no general rule to select $N$ and the number of Chebyshev points $q_{k_{1}}, q_{k_{2}}, q_{x_{1}}, q_{x_{2}}$ (recall that $r_{\varepsilon}=q_{k_{1}} q_{k_{2}}$ or $r_{\varepsilon}=$ $\left.q_{x_{1}} q_{x_{2}}\right)$. For numerical implementation, these parameters are tuned to achieve the best efficiency and accuracy.

\section{NUMERICAL EXAMPLES}

\section{D synthetic data}

We start with a simple 2D example. Figure 2 shows a synthetic CMP gather sampled on $N_{t} \times N_{h}=1000^{2}$. Figure 3 shows the result by the fast butterfly algorithm obtained on $N_{\tau} \times N_{p}=$ $1000^{2}$. For this problem, our method provides a reasonable result with $N=32, q_{k_{1}}=q_{x_{1}}=7, q_{k_{2}}=q_{x_{2}}=5$ in only 1 second of CPU time, while the traditional velocity scan takes about $60 \mathrm{~s}$, whose result is also shown here for comparison (Figure 4).

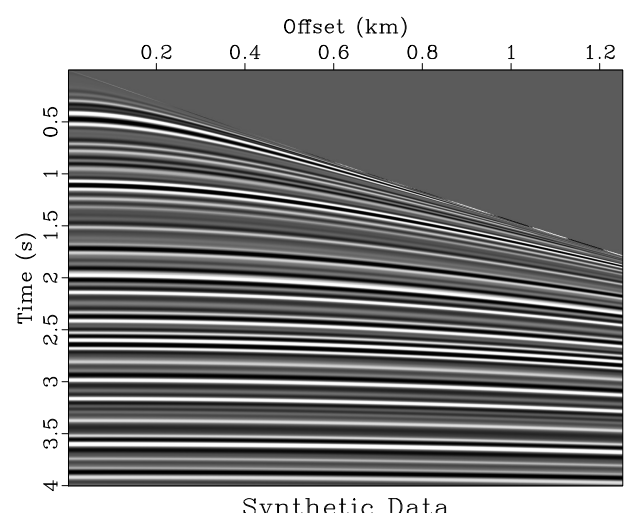

Figure 2: 2D synthetic CMP gather, $N_{t} \times N_{h}=1000^{2}$.



Figure 3: Output of the fast butterfly algorithm, $N_{\tau} \times N_{p}=$ $1000^{2}, N=32, q_{k_{1}}=q_{x_{1}}=7, q_{k_{2}}=q_{x_{2}}=5$; CPU time: $\mathbf{1 . 2 6}$ s.

\section{D field data}

We now consider a real 2D seismic gather in Figure 5 The sampling sizes are $N_{t}=N_{\tau}=1500, N_{h}=240$, and $N_{p}=2000$. Parameters are chosen as $N=128, q_{k_{1}}=q_{x_{1}}=7, q_{k_{2}}=q_{x_{2}}=$ 5. Although the small $N_{h}$ makes these data not very suitable for testing the fast algorithm, our method $(\sim 7 \mathrm{~s})$ still outperforms the velocity scan $(\sim 43 \mathrm{~s})$ with acceptable accuracy (Figure 6).

\section{D synthetic data}

Since the above discussion does not require the input data to be uniform, our algorithm can be easily extended to handle the 


\section{Fast Radon Transform}

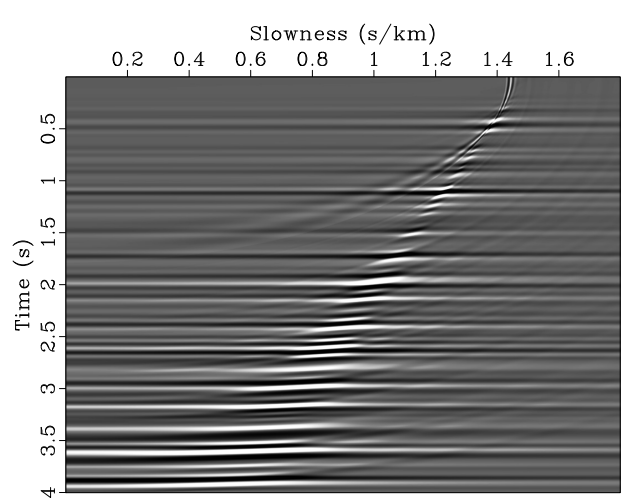

Figure 4: Output of the velocity scan, $N_{\tau} \times N_{p}=1000^{2}$; CPU time: $61.36 \mathrm{~s}$.

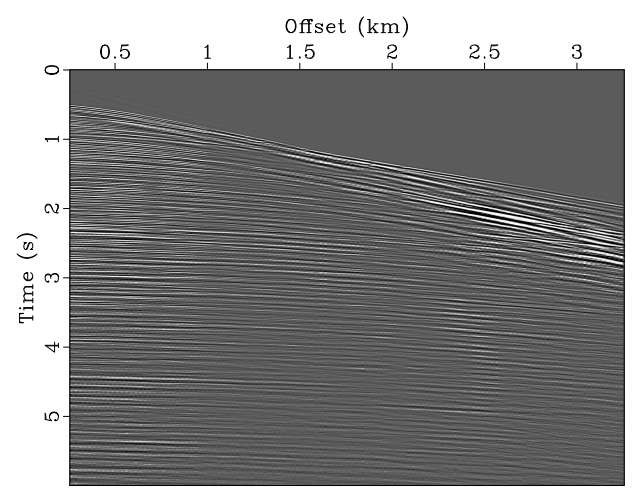

Field Data

Figure 5: 2D real CMP gather, $N_{t}=1500, N_{h}=240$.

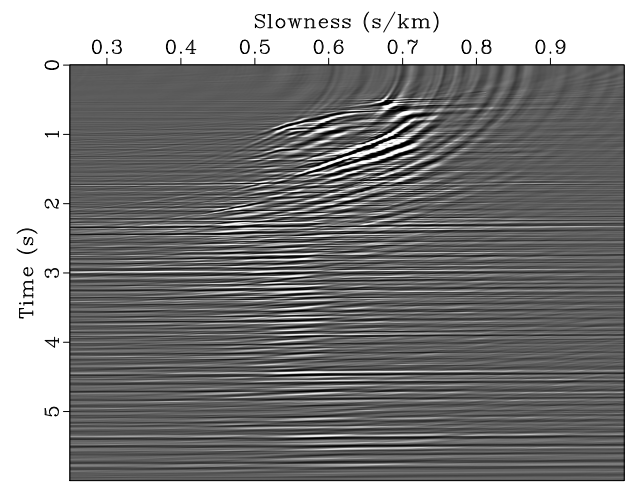

Figure 6: Output of the fast butterfly algorithm, $N_{\tau}=1500$, $N_{p}=2000, N=128, q_{k_{1}}=q_{x_{1}}=7, q_{k_{2}}=q_{x_{2}}=5$; CPU time: $\mathbf{7 . 2 3} \mathrm{s}$ (ref: CPU time of velocity scan is $\mathbf{4 2 . 5 8} \mathbf{~ s}$ ). following problem:

$$
(R d)(\tau, p)=\iint d\left(\sqrt{\tau^{2}+p^{2}\left(h_{1}^{2}+h_{2}^{2}\right)}, h_{1}, h_{2}\right) d h_{1} d h_{2},
$$

where $d\left(t, h_{1}, h_{2}\right)$ is a function in $3 \mathrm{D}$ rather than $2 \mathrm{D}$. All we need is to introduce a new variable $h=\sqrt{h_{1}^{2}+h_{2}^{2}}$, and reorder the values $d\left(t, h_{1}, h_{2}\right)$ according to $h$. Figure 7 is such synthetic data sampled on $N_{t} \times N_{h_{1}} N_{h_{2}}=1000 \times 128^{2}$. The output is obtained for $N_{\tau} \times N_{p}=1000 \times 512$. The fast algorithm (Figure 8) runs in only $7 \mathrm{~s}$ for $N=64, q_{k_{1}}=q_{x_{1}}=5, q_{k_{2}}=q_{x_{2}}=3$, while the velocity scan takes more than $500 \mathrm{~s}$.



Figure 7: 3D synthetic CMP gather, $N_{t}=1000, N_{h_{1}}=N_{h_{2}}=$ 128.

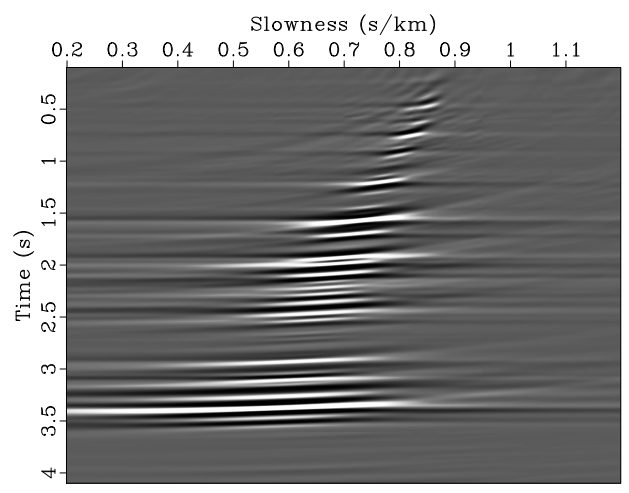

Figure 8: Output of the fast butterfly algorithm, $N_{\tau}=1000$, $N_{p}=512, N=64, q_{k_{1}}=q_{x_{1}}=5, q_{k_{2}}=q_{x_{2}}=3$; CPU time: $\mathbf{7 . 3 4} \mathrm{s}$ (ref: CPU time of velocity scan is $\mathbf{5 1 5 . 2 5} \mathbf{~ s}$ ).

\section{CONCLUSIONS}

We constructed a fast butterfly algorithm for the hyperbolic Radon transform. Compared with the time-consuming integration in the time domain, our method runs in only $O\left(N^{2} \log N\right)$ operations, where $N$ is representative of the number of points in either dimension of data space or model space. An ongoing work is to study the performance of this fast solver for the sparse iterative inversion of the Radon transform in application to multiple attenuation. 


\section{Fast Radon Transform}

\section{REFERENCES}

Candès, E., L. Demanet, and L. Ying, 2009, A fast butterfly algorithm for the computation of Fourier integral operators: Multiscale Model. Simul., 7, 1727-1750.

Cary, P. W., 1998, The simplest discrete Radon transform: SEG Annual Meeting, September 13 - 18, 1998, New Orleans, Louisiana. Foster, D. J., and C. C. Mosher, 1992, Suppression of multiple reflections using the Radon transform: Geophysics, 57, 386-395.

Gardner, G. H. F., and L. Lu, eds., 1991, Slant-stack processing: Society of Exploration Geophysicists. Issue 14 of Geophysics reprint series.

Hampson, D., 1986, Inverse velocity stacking for multiple elimination: J. Can. Soc. Expl. Geophys., 22, 44-55.

Hargreaves, N., B. verWest, R. Wombell, and D. Trad, 2003, Multiple attenuation using an apex-shifted Radon transform: EAGE 65th Conference and Exhibition, June 2 -5, 2003, Stavanger, Norway.

Herrmann, P., T. Mojesky, M. Magesan, and P. Hugonnet, 2000, De-aliased, high-resolution Radon transforms: SEG Annual Meeting, August 6 - 11, 2000, Calgary, Alberta.

Michielssen, E., and A. Boag, 1996, A multilevel matrix decomposition algorithm for analyzing scattering from large structures: IEEE Trans. Antennas and Propagation, 44, 1086-1093.

Moore, I., and C. Kostov, 2002, Stable, efficient, high-resolution Radon transforms: EAGE 64th Conference and Exhibition, May 27 - 30, 2002, Florence, Italy.

O'Neil, M., and V. Rokhlin, 2007, A new class of analysis-based fast transforms: Technical report YALEU/DCS/TR-1384, Yale University, New Haven, CT.

Radon, J., 1917, Über die bestimmung von funktionen durch ihre integralwerte längs gewisser mannigfaltigkeiten: Berichte über die Verhandlungen der Sächsische Akademie der Wissenschaften (Reports on the proceedings of the Saxony Academy of Science), 69, 262-277.

Sacchi, M., 1996, A bidiagonalization procedure for the inversion of time-variant velocity stack operator: CDSST report, 73-92.

Thorson, J. R., and J. F. Claerbout, 1985, Velocity-stack and slant-stack stochastic inversion: Geophysics, 50, $2727-2741$.

Trad, D., 2003, Interpolation and multiple attenuation with migration operators: Geophysics, 68, 2043-2054.

Trad, D., T. Ulrych, and M. Sacchi, 2002, Accurate interpolation with high resolution time-variant Radon transforms: Geophysics, 67, 644-656.

Yilmaz, O., 1989, Velocity-stack processing: Geophysical Prospecting, 37, 357-382. 